\title{
Hydrodynamics of membranes: the bilayer aspect and adhesion
}

\author{
Udo Seifert \\ Institut für Festkörperforschung, Forschungszentrum Jülich, 52425 Jülich (Germany) \\ and \\ Stephen A. Langer \\ Department of Physics, Simon Fraser University, Burnaby, British Columbia V5A 156 (Canada)
}

(Received 15 June 1993; accepted in revised form 4 October 1993)

\begin{abstract}
We review recent work on the dynamics of membranes. First, we discuss the viscous modes of a free bilayer membrane, taking into account the coupling between bending and the local density of the two monolayers. Apart from important corrections to the conventional bending mode, we obtain a second slow mode which is essentially a fluctuation in the density difference of the two monolayers, damped by intermonolayer friction. Estimates for a stack of membranes show reasonable agreement with a recent spin-echo study of membrane undulations. Second, we discuss the dispersion relation for both fluid and polymerized membranes bound to a substrate by an attractive potential. The interplay between the hydrodynamic damping by the surrounding liquid and the restoring force in the binding potential leads to a $q^{2}$ dependence of the damping rate $\gamma$ at small wavevector $q$. With increasing $q$, various crossovers can occur, including the possibility of nonmonotonic damping in which $\gamma$ decreases with $q$ as $\sim 1 / q$.
\end{abstract}

Keywords: Membrane hydrodynamics; Bending modes; Viscous damping; Adhesion

\section{Introduction}

The traditional model for the dynamics of fluid phospholipid membranes treats them as a single incompressible sheet with bending rigidity, whose fluctuations are damped by viscous friction in the surrounding liquid (for reviews on membranes, see c.g. ref. [1]) [2,3]. In this contribution, we briefly review recent theoretical results about two extensions of this simple picture.

First, in section 2 we review the standard description of monolayer membranes. In section 3 we discuss the dynamics of a bilayer membrane taking into account the fact that the membrane consists of a pair of slightly compressible monolayers bound tightly together [4]. This bilayer structure implies that bending a membrane necessarily leads to a stretching of one monolayer and a compression of the other. 
Since the membrane is fluid, density inhomogeneities can relax within each monolayer by lateral lipid flow. For the investigation of static equilibrium phenomena, one can therefore assume that the lipid density within each monolayer is homogeneous. The only effect of the bilayer structure is to add a global term to the energy, the area-difference elasticity [5-7], which is important for calculating the phase diagram of vesicle shapes. Evans and co-workers [8] recently stressed that for the dynamics of conformational changes of membranes the coupling between bending and relative compression is crucial, and demonstrated this in the analysis of a tether formation experiment. We analyze the much simpler but paradigmatic case of the dynamical equilibrium fluctuations of an almost planar bilayer where the lateral lipid flow is also subject to friction between the slipping chains of the two opposing monolayers. This provides an additional dissipative mechanism which dominates the dynamics on length scales below one micron.

Since a direct verification of these results on a single membrane will be difficult, in section 4 we discuss the corresponding modes for a multilamellar membrane stack. These results can indeed be compared with a recent experiment on DMPC multilayers [9].

Our second topic concerns the dynamics of a bound planar membrane, which is a paradigmatic model for the experimentally accessible situation of an adhering giant vesicle [10-12]. Through a new technique, reflection interference microscopy [13-15], which allows quite precise measurements of the separation of a membrane from a substrate, it has become possible to measure dynamical fluctuations of such a bound membrane. In section 5, we discuss the dispersion relation for the bending modes of a bound fluid membrane which is governed by the interplay of three relevant length scales, the wavelength, the separation from the substrate, and the correlation length of the membrane [16]. We close in section 6 with the dispersion relation for a bound polymerized membrane.

\section{The traditional picture}

We first cast the standard treatment of the dynamics of a membrane [2,3] in a form which facilitates a qualitative discussion of the refinements presented in subsequent sections.

In the Monge representation, an almost planar membrane is parametrized by a function $h(x, y)$. The bending energy stored in a displacement $h(x, y)=h_{q}\left(\mathrm{e}^{i q x}+\right.$ c.c. $)$ described by a single Fourier amplitude $h_{q}$ is

$F=\frac{1}{2} \kappa q^{4} h_{q} h_{q}^{*} \equiv \frac{1}{2} E(q) h_{q} h_{q}^{*}$,

where $\kappa$ is the bending rigidity and $E(q)$ will be called the "energy" of this mode. Such a deformation leads to a restoring force $\delta F / \delta h_{q}^{*}$ which acts on the surrounding incompressible liquid. This liquid can be described within linearized hydrodynamics by the Stokes approximation

$\boldsymbol{\nabla} \cdot \boldsymbol{v}=0 \quad$ and $\quad \eta \nabla^{2} v=\nabla p \quad$ for $\quad z \neq 0$,

where $z$ is the coordinate perpendicular to the membrane at $z=0, p$ is the pressure in the liquid, and $\eta$ is its viscosity. At the membrane, the normal forces have to balance, which implies

$-T_{z z}^{+}+T_{z z}^{-}=-\delta F / \delta h_{q}$,

where $T_{z z}^{ \pm}$is the $(z, z)$ component of the liquid stress tensor

$T_{i j} \equiv-p \delta_{i j}+\eta\left(\partial_{i} v_{j}+\partial_{j} v_{i}\right)$

at $z= \pm 0$. 
These equations are solved with the following boundary conditions:

(i) vanishing liquid velocity as $z \rightarrow \pm \infty$,

(ii) continuity of the normal liquid velocity at $z=0$,

(iii) impermeability of the membrane, i.e. $v_{z}(z= \pm 0)=\partial_{t} h_{q}$,

(iv) continuity of the in-plane liquid velocity at $z=0$,

(v) incompressibility of the membrane, which requires that the in-plane divergence of $\mathbf{v}$ vanishes at $z=0$.

After eliminating the velocity field, one obtains the equation of motion for the membrane:

$\partial_{t} h_{q}=-\Gamma(q) \delta F / \delta h_{q}^{*}=-\Gamma(q) E(q) h_{q}$.

Here $\Gamma(q)=1 / 4 \eta q$ is the kinetic coefficient which reflects the long-range character of the hydrodynamic damping. In the solution to eq. (5), $h_{q}(t)=h_{q}(0) \mathrm{e}^{-\gamma(q) t}$, the damping rate $\gamma(q)$ is easily identified as

$\gamma(q)=\Gamma(q) E(q)$,

leading to $\gamma=\kappa q^{3} / 4 \eta$ in the traditional model. The form (6) of the damping rate as a product [17] of a kinetic coefficient which contains the dissipation and an energy which contains the driving force will persist in the more refined model discussed below.

\section{Dispersion relation for a bilayer membrane}

The model just described ignores the fact that a membrane consists of a pair of slightly compressible monolayers bound tightly together. To discuss the dynamical implications of this structure, we first have to introduce two densities $\phi^{ \pm}$and $\psi^{ \pm}$for the upper (+) and lower (-) monolayers (see fig. 1). $\phi^{ \pm}$ describes the density of lipids at the neutral surface of each monolayer. When the membrane is curved, the densities $\psi^{ \pm}$projected onto the midsurface of the bilayer will differ from the densities $\phi^{ \pm}$on the neutral surfaces of the monolayers. To lowest order in $d H$ these two densities are related by $\phi^{ \pm} \approx \psi^{ \pm}(1$

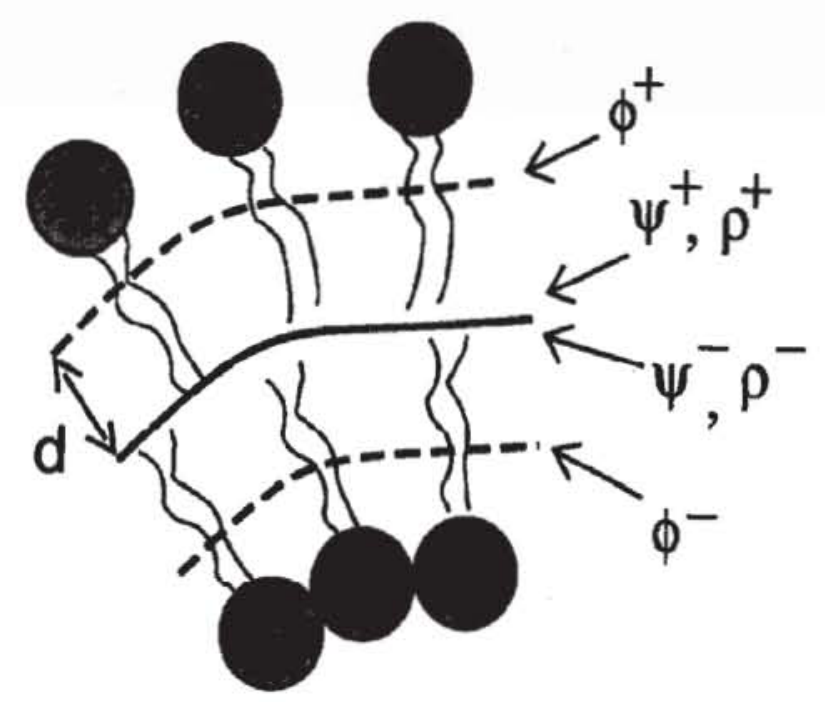

Fig. 1. Schematic geometry of a bilayer membrane. The circles with squiggly tails represent the lipid molecules. The dashed lines are the neutral surfaces of the monolayers, on which the densities $\phi^{ \pm}$are defined. The dark solid line is the midsurface of the bilayer, on which the projected densities $\psi^{ \pm}$and the scaled projected densities $\rho^{ \pm}$are defined. 
$\pm 2 d H$ ), where $H$ is the mean curvature of the bilayer and $d$ the distance between the midsurface of the bilayer and the neutral surface of a monolayer. The elastic energy density of each monolayer is given by $\left(\frac{1}{2} k\right)\left(\phi^{ \pm} / \phi_{0}-1\right)^{2} \approx\left(\frac{1}{2} k\right)\left(\rho^{ \pm} \pm 2 d H\right)^{2}$, where $\rho^{ \pm} \equiv\left(\psi^{ \pm} / \phi_{0}-1\right)$ is the scaled deviation of the projected density from its equilibrium value $\phi_{0}$ for a flat membrane. $k$ is the area compression modulus of a monolayer. Thus the continuum free energy, $F$, for the entire membrane reads

$F=\int \mathrm{d} A\left\{\frac{1}{2} \kappa(2 H)^{2}+\frac{1}{2} k\left[\left(\rho^{+}+2 d H\right)^{2}+\left(\rho^{-}-2 d H\right)^{2}\right]\right\}$.

The first term arises from the bending energy of each monolayer. (We have implicitly assumed that the monolayers are symmetric and have spontaneous curvature $C_{0}^{(\mathrm{m})} \ll d^{-1}$.) As written, $F$ is a functional of the membrane shape and the two densities $\rho^{ \pm}$.

As above, we consider a plane wave in the $x$ direction, $h(x, y) \equiv h_{q} \mathrm{e}^{i q x}+$ c.c. and $\rho^{ \pm}(x, y) \equiv\left(\bar{\rho}_{q} \pm\right.$ $\left.\rho_{q}\right) \mathrm{e}^{i q x}+$ c.c., with a density difference $\rho_{q} \equiv \frac{1}{2}\left(\rho_{q}^{+}-\rho_{q}^{-}\right)$, and an average density $\bar{\rho}_{q} \equiv \frac{1}{2}\left(\rho_{q}^{+}+\rho_{q}^{-}\right)$. With $2 H=\nabla^{2} h(x, y)$ this leads to the energy

$F=\frac{1}{2}\left(h_{q}, \rho_{q}\right)\left(\begin{array}{cc}\tilde{\kappa} q^{4} & -2 k d q^{2} \\ -2 k d q^{2} & 2 k\end{array}\right)\left(\begin{array}{c}h_{q}^{*} \\ \rho_{q}^{*}\end{array}\right) \equiv \frac{1}{2}\left(h_{q}, \rho_{q}\right) \mathbf{E}(q)\left(\begin{array}{c}h_{q}^{*} \\ \rho_{q}^{*}\end{array}\right)$,

where $\mathbf{E}$ denotes the energy matrix which couples the density difference to the shape. We omit a term quadratic in the mean density $\bar{\rho}_{q}$ since it does not couple to the shape at this level of approximation. In eq. (8), $\tilde{\kappa}=\kappa+2 d^{2} k$ is a renormalized bending rigidity which includes the effect of the elastic stretching and compression. Note that if (8) is minimized with respect to $\rho_{q}$ one recovers the energy (1) of the traditional model. This shows that the traditional model implicitly assumes that the densities in each monolayer adjust optimally to the shape. Obviously this can only be the case if the density relaxation is faster than the bending modes. It turns out that this assumption does not hold true for all wavelengths.

The compressible bilayer description requires that we balance not only the normal forces given by (3) but also the forces within each monolayer. This balance reads

$-\bar{\nabla} \sigma^{ \pm} \pm T_{x z}^{ \pm}+\mu \tilde{\nabla}^{2} \tilde{v}^{ \pm} \mp b\left(\tilde{v}^{+}-\tilde{v}^{-}\right)=0$,

where the tilde refers to two-dimensional quantities. The four force densities in (9) are (i) the (in-plane) gradient of the surface pressure $-\tilde{\nabla} \sigma^{ \pm}=-\tilde{\nabla}\left(\delta F / \delta \rho^{ \pm *}\right)$; (ii) the traction of the surrounding fluid, given by the liquid stress tensor; (iii) the viscous damping within each monolayer, where $\mu$ is the monolayer (surface) viscosity and $\tilde{v}^{ \pm}$is the velocity of the lipid flow within each monolayer; and (iv) the friction between the two monolayers, with the phenomenological friction coefficient $b$.

The densities $\rho \pm$ obey equations of continuity $\partial_{t} \rho^{ \pm} \approx-\tilde{\nabla} \cdot \tilde{v}^{+}$to lowest order in the small quantities $\rho^{ \pm}$and $\nu$.

While the boundary conditions (i)-(iii) listed in section II still apply, we now also require continuity between the in-plane velocity of the fluid at $z= \pm 0$ and the velocity of the adjacent monolayer. Solving the hydrodynamic equations with these boundary conditions leads to the equation of motion for the coupled height and density difference variables:

$$
\frac{\partial}{\partial t}\left(\begin{array}{l}
h_{q} \\
\rho_{q}
\end{array}\right)=-\boldsymbol{\Gamma}(q) \mathbf{E}(q)\left(\begin{array}{l}
h_{q} \\
\rho_{q}
\end{array}\right) \text {. }
$$

The matrix of kinetic coefficients

$\boldsymbol{\Gamma}(q) \equiv\left(\begin{array}{cc}1 / 4 \eta q & 0 \\ 0 & \frac{q^{2}}{2\left(2 b+2 \eta q+\mu q^{2}\right)}\end{array}\right)$ 


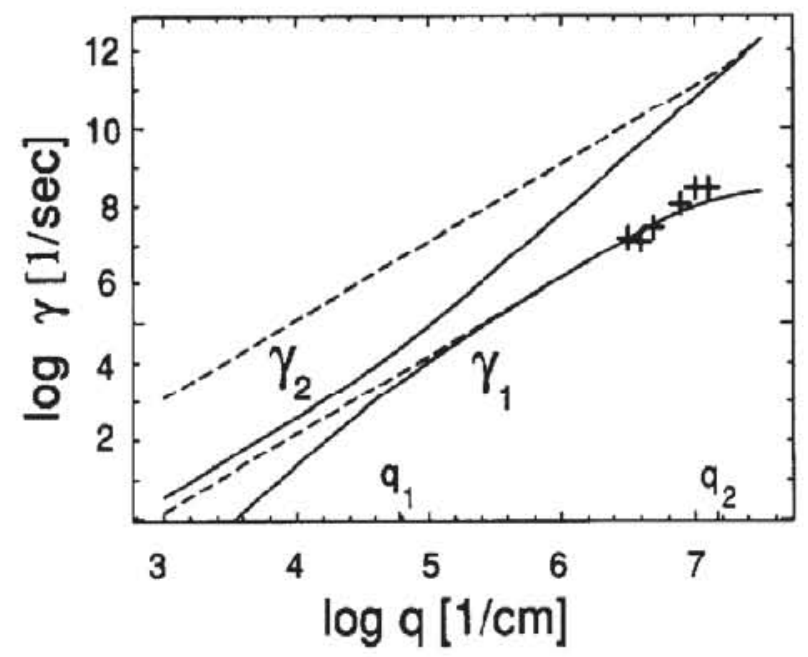

Fig. 2. Dispersion relations for a single bilayer (full line) and a stack of bilayers (dashed line) for $k=10^{-12} \mathrm{erg}, k=70 \mathrm{erg} / \mathrm{cm}^{2}$, $d=1 \mathrm{~nm}, \eta=0.01 \mathrm{erg} \mathrm{s} / \mathrm{cm}^{3}, \mu=10^{-7} \mathrm{erg} \mathrm{s} / \mathrm{cm}^{2}, b=10^{7} \mathrm{erg} \mathrm{s} / \mathrm{cm}^{4}$; and $l=1 \mathrm{~nm}$ for the stack. The marks are experimental data [9].

is diagonal with the same $\left(h_{q}, h_{q}^{*}\right)$ entry as the traditional model. For small $q$, the dissipation for the density difference mode is provided by the intermonolayer friction with coefficient $b$. It has a $q^{2}$ dependence because densities are conserved quantities.

The products $\Gamma(q) \mathbf{E}(q)$ has two eigenvalues $\gamma_{1,2}(q)$, which give the dispersion relation shown in fig. 2 . There are three regimes [4] separated by the crossover wavevectors $q_{1} \equiv 2 \eta k / b \tilde{\kappa}$ and $q_{2} \equiv \sqrt{2 b / \mu}$ :

$\gamma_{1} \approx\left\{\begin{array}{ll}\frac{\kappa}{4 \eta} q^{3}, & q \ll q_{1}, \\ \frac{k}{2 b} \frac{\kappa}{\tilde{\kappa}} q^{2}, & q_{1} \ll q \ll q_{2}, \\ \frac{k}{\mu} \frac{\kappa}{\tilde{\kappa}}, & q_{2} \ll q,\end{array} \quad \gamma_{2} \approx \begin{cases}\frac{k}{2 b} q^{2}, & q \ll q_{1}, \\ \frac{\tilde{\kappa}}{4 \eta} q^{3}, & q_{1} \ll q .\end{cases}\right.$

For small $q, \gamma_{1}$ corresponds to the usual hydrodynamically damped bending mode and $\gamma_{2}$ is the damping rate of a new "slipping" mode, a density difference fluctuation damped by the intermonolayer friction. As shown in fig. 2, the modes mix and the damping rates deviate significantly from their asymptotic low $q$ behavior above the crossover wavevector $q_{1}$. For $q \gg q_{1}, \gamma_{2}$ becomes the damping rate of the (predominantly) bending mode, with an effective bending rigidity $\tilde{\kappa}$. The effective high-frequency rigidity differs from the low-frequency rigidity $\kappa$ because the densities cannot responds quickly to changes in shape. In this regime, the slower rate $\gamma_{1}$ is predominantly due to slipping. $\gamma_{1}$ exhibits a second crossover at $q_{2}$ $\equiv \sqrt{2 b / \mu}$ where the main dissipative mechanism changes from intermonolayer friction to monolayer surface viscosity.

To estimate the crossover wavelengths, we use typical parameter values for phospholipid bilayers: the bending rigidity $\kappa=10^{-12} \mathrm{erg}$, the viscosity of water $\eta=10^{-2} \mathrm{erg} \mathrm{s} / \mathrm{cm}^{3}$, the monolayer area expansivity $k=10^{2} \mathrm{erg} / \mathrm{cm}^{2}$, and half the monolayer thickness $d=1 \mathrm{~nm}$. The remaining model parameters, the intermonolayer friction coefficient $b$ and the monolayer viscosity $\mu$, are not yet well studied. Measurements of the diffusion constants in free and supported bilayers by microfluorescence techniques [18] lead to the values $\mu \approx 10^{-7}-10^{-6} \mathrm{erg} \mathrm{s} / \mathrm{cm}^{2}$ and $b \simeq 10^{7}-10^{8} \mathrm{erg} \mathrm{s} / \mathrm{cm}^{4}$, while the friction coefficient value inferred from the tether formation experiment [8] is $b \simeq 5 \times 10^{7} \mathrm{erg} \mathrm{s} / \mathrm{cm}^{4}$. For $b=10^{7} \mathrm{erg} \mathrm{s} / \mathrm{cm}^{4}$, we 
find for the first crossover $q_{1} \approx 10^{5} / \mathrm{cm}$, corresponding to a wavelength in the micron range. Therefore, bilayer corrections to the dispersion relation for the bending mode become important on length scales of microns and below. In particular, for $q>q_{1}$, the slowest mode of the bilayer becomes the new slipping mode. For the second crossover, relevant only to the slower mode, we find $q_{2} \simeq 10^{7} / \mathrm{cm}$, i.e. a wavelength of several times the membrane thickness.

Direct measurements of the wavelength dependent relaxation times of single bilayers by video microscopy of vesicle fluctuations have been restricted to wavelengths larger than half a micron $[19,20]$. Even though we expect to see deviations from the asymptotic low $q$ behavior in this region, experimental limitations as well as additional complications due to area and volume constraints [21] will make detection nontrivial.

\section{Viscous modes of a membrane stack}

A more promising technique to verify the effect of the bilayer structure in membrane dynamics might be dynamical light scattering on multilayer systems [22]. For a stack of swollen membranes and a wavevector parallel to the sheets, the calculation of the dispersion relation for the collective undulation mode using the same force balance and boundary conditions at every membrane in a stack with repeat distance $2 l$ is absolutely straightforward. We again find two modes, whose dispersion relation is shown in fig. 2. For small $q$, both modes have a quadratic dispersion,

$$
\gamma_{1} \approx \frac{k}{2 b} \frac{\kappa}{\tilde{\kappa}} q^{2}, \quad \text { and } \quad \gamma_{2} \approx \frac{\tilde{\kappa}}{2 \eta l} q^{2} .
$$

Here, $\gamma_{2}$ corresponds to the undulation mode of a two components smectic [23], which crosses over into the single layer result for $q \sim 1 / l$. The damping rate of the second slow mode, $\gamma_{1}$, has the same dispersion as the single bilayer in the intermediate $q$ regime.

The dispersion relation for $\gamma_{1}$ in the multilayer system can be compared with a recent spin echo study of the undulations of swollen phospholipid multilayers [9]. In fig. 2, we show these data obtained in the $q$ range $0.3-1.2 \times 10^{7} / \mathrm{cm}$. Even though the agreement is good, more work will clearly be needed to prove that this measurements corresponds to the mode discussed in this paper. A crucial experimental test would be to measure the damping as a function of the repeat distance $2 l$. If the measured dispersion is indeed that of the frictional mode, it should be independent of the repeat distance, since the main dissipation for this mode occurs within the bilayer rather than in the liquid.

\section{Dynamics of a bound fluid membrane}

We now turn to a membrane interacting with a substrate through a potential $V(l)$. For simplicity, we describe the membrane within the traditional model as an incompressible sheet, thus focusing on the new effects arising from the interaction with the substrate. The energy $F$ of a membrane at $l(x, y)$ in a potential $V(l)$ is $[24,25]$

$F=\int \mathrm{d} x \mathrm{~d} y\left[\frac{1}{2} \kappa\left(\nabla^{2} l\right)^{2}+V(l)\right] \approx \frac{1}{2} \kappa \int \mathrm{d} x \mathrm{~d} y\left[\left(\nabla^{2} h\right)^{2}+\xi^{-4} h^{2}\right]$,

using a harmonic approximation for fluctuations $h(x, y) \equiv l(x, y)-l_{0}$ around the minimum of the 
potential at $l=l_{0}$, and defining the parallel correlation length $\xi \equiv\left(\kappa / \mathrm{d}^{2} V /\left.\mathrm{d} l^{2}\right|_{l=l_{0}}\right)^{1 / 4}$. For a plane wave bending mode with wavevector $q$ and amplitude $h_{q}$,

$F \equiv \frac{1}{2} E(q, \xi) h_{q} h_{q}^{*}=\frac{1}{2} \kappa\left(q^{4}+\xi^{-4}\right) h_{q} h_{q}^{*}$.

Thus, unlike the free membrane, at small $q$ the energy $E(q, \xi)$ is dominated by the $\xi^{-4}$ term arising from the confining potential.

The equations for the surrounding liquid and the normal force balance are as in section 2, with $z=0$ replaced by $z=l_{0}$. While the boundary conditions (ii) through (v) still hold, the presence of the wall requires that the velocity field of the liquid vanishes at the substrate $(z=0)$. Assuming plane waves, and solving the hydrodynamic equations, one obtains a damping rate of the form [16]

$\gamma\left(q, l_{0}, \xi\right)=\Gamma\left(q, l_{0}\right) E(q, \xi)$

with the energy $E(q, \xi)$ given by (15) and the kinetic coefficient

$\Gamma\left(q, l_{0}\right) \equiv \frac{1}{2 \eta q} \frac{\sinh ^{2}\left(q l_{0}\right)-\left(q l_{0}\right)^{2}}{\sinh ^{2}\left(q l_{0}\right)-\left(q l_{0}\right)^{2}+\sinh \left(q l_{0}\right) \cosh \left(q l_{0}\right)+\left(q l_{0}\right)} \rightarrow \begin{cases}l_{0}^{3} q^{2} / 12 \eta, & q \ll 1 / l_{0} \\ 1 / 4 \eta q, & q \gg 1 / l_{0} .\end{cases}$

The asymptotic behavior of $\Gamma\left(q, l_{0}\right)$ can easily be understood as follows: for $q \geqslant 1 / l_{0}$, the distortion of the velocity field in the liquid decays so fast that it does not feel the presence of the substrate. $\Gamma$ is then given by its form for a free membrane. For $q \ll 1 / l_{0}, \Gamma$ has the usual quadratic $q$ behavior for conserved quantities, the conserved quantity here being the volume of liquid between the membrane and the substrate.

Due to the confining potential, the small $q$ behavior of the damping rate,

$\gamma \approx \kappa l_{0}^{3} q^{2} / 12 \eta \xi^{4}, \quad q \ll \min \left[1 / l_{0}, 1 / \xi\right]$,

differs from the $\gamma \sim q^{6}$ behavior Brochard and Lennon [3] derived for two parallel membranes with no potential, i.e. formally for $\xi=\infty$. For the dispersion relation at intermediate wavevectors $q$ there are two cases, depending on the relative sizes of the crossover length scales for the kinetic coefficient $\left(l_{0}\right)$ and the energy $(\xi)$. (i) For $l_{0} \ll \xi$, (16) and (17) imply the intermediate behavior

$\gamma \approx \kappa l_{0}^{3} q^{6} / 12 \eta, \quad 1 / \xi \ll q \ll 1 / l_{0}$,

which will be called monotonic damping. In this case, the potential is irrelevant, and we indeed recover the low- $q$ limit of Brochard and Lennon. (ii) For $\xi<l_{0}$, the $q$ dependency becomes

$\gamma \approx \kappa / 4 \eta \xi^{4} q, \quad 1 / l_{0} \ll q \ll 1 / \xi$.

In this case, the damping rate decreases with increasing wavevector. This will be referred to as nonmonotonic damping. It arises from the fact that the potential confines the mean square amplitudes $\left\langle h_{q}^{2}\right\rangle$ to the value $\left\langle h_{q}^{2}\right\rangle \approx T \xi^{4} / \kappa$ independently of $q$, while the hydrodynamic damping becomes less effective with increasing $q$. Finally, for large $q \gg \max \left[1 / l_{0}, 1 / \xi\right]$, we recover the free damping rate $\gamma \approx \kappa q^{3} / 4 \eta$.

Although the potential $V(l)$ determines both the lengths $l_{0}$ and $\xi$, whether it leads to monotonic or nonmonotonic damping depends, in addition, on the strength of nonharmonic fluctuations, which can enhance the repulsion of the membrane from the substrate. According to Lipowsky's general classification [26] of adhesion potentials based merely on static properties, three regimes for the influence of fluctuations have to be distinguished: (i) the mean field regime, (ii) the weak fluctuation regime, and (iii) the strong fluctuation regime. Using an illustrative example, we now discuss the dynamical behavior in these three cases by relating them to the two different dynamical regimes described above. 
The mean field regime: consider a charged membrane pushed by an osmotic pressure $p$ towards a substrate. In weak electrolytes, where the screening length is large compared to $l_{0}$, fluctuations beyond the harmonic level can safely be ignored. The potential is then [25]

$V(l)=A / l+p l$,

where $A \equiv\left(\pi T / 2 l_{\mathrm{B}}\right)[27]$. Here, $l_{\mathrm{B}} \approx 0.7 \mathrm{~nm}$ is the Bjerrum length in water and $T$ the temperature (with Boltzmann's constant set to unity). One immediately gets $l_{0}=(A / p)^{1 / 2}$ and $\xi=(\kappa / 2 A)^{1 / 4} l_{0}^{3 / 4}$. In the small $q$ range, this implies that the damping rate $\gamma \approx(A / 6 \eta) q^{2}$ does not depend on the mean separation $l_{0}$ for this potential. For an estimate of the typical time scales involved, we find $\gamma \approx\left(10^{-5}\right.$ $\left.\mathrm{cm}^{2} / \mathrm{s}\right) q^{2}$, using the typical values $\kappa \simeq 10^{-12} \mathrm{erg} \simeq 25 T$ for phospholipids and $\eta=10^{-2} \mathrm{erg} \mathrm{s} / \mathrm{cm}^{3}$ for water. For a wavelength $\lambda=1 \mu \mathrm{m}=2 \pi / q$, this becomes $\gamma \simeq 6 \times 10^{4} / \mathrm{s}$ which is too fast to be visible by video microscopy but will be accessible to dynamical light scattering. The criterion $l_{0} \gg \xi$, implying nonmonotonic damping, is met whenever $l_{0} \gg \kappa l_{\mathrm{B}} / \pi T \simeq 6 \mathrm{~nm}$. Thus, we predict a damping rate which decreases with $q$ in the range $1 / l_{0} \ll q \ll 1 / \xi$ for unscreened electrostatic interactions (and, quite generally, for adhesion potentials which belong to the mean field regime). For $p \rightarrow 0$, which corresponds to the unbinding transition $[24,28], l_{0}$ becomes much larger than $\xi$. Thus, the two crossover wavevectors, $1 / l_{0}$ and $1 / \xi$, in (20) scale differently at the unbinding transition and the intermediate anomalous behavior of $\gamma$ should, in principle, be clearly detectable.

The weak fluctuation regime: In stronger electrolytes, the electrostatic repulsion is screened and becomes exponential in $l$. Nonharmonic fluctuations can then no longer be neglected. In a self-consistent way they can be included by adding a steric interaction [29] $V_{\mathrm{FL}}=c\left(T^{2} / \kappa\right) / l^{2}$ to the effective potential. $c$ is a numerical coefficient of order one. Ignoring the electrostatic repulsion for simplicity, the total potential now reads

$V(l)=c T^{2} / \kappa l^{2}+p l$.

The equilibrium separation is given by $l_{0}=(2 c / p)^{1 / 3} T^{2 / 3} / \kappa^{1 / 3}$ and the relation between the correlation length $\xi=(\kappa / T)^{1 / 2} l_{0} /(6 c)^{1 / 4} \simeq 3 l_{0}$ and $l_{0}$ becomes independent of the amplitude $p$ of the linear attractive potential. Since $\xi>l_{0}$, the weak fluctuation regime will always be governed by monotonic damping. Note that even for $p \rightarrow 0$ the intermediate behavior, $\gamma \sim q^{6}$, will be confined to the rather narrow interval $1 / 3 l_{0} \leqq q \leqq 1 / l_{0}$. For an estimate of the typical damping rates, assuming small $q$ and taking $c=1$, we get $\gamma \approx\left(c T^{2} / 2 \eta \kappa\right) q^{2} / l_{0} \simeq\left(10^{-13} \mathrm{~cm}^{3} / \mathrm{s}\right) q^{2} / l_{0}$. For $l_{0}=50 \mathrm{~nm}$ and $\lambda=2 \mu \mathrm{m}$, one obtains $\gamma \approx 10 / \mathrm{s}$, which is below video frequency. Thus, these fluctuations will be accessible to micro-optical techniques [13-15].

The strong fluctuation regime: if both the attractive as well as the repulsive potential become short ranged the nonharmonic fluctuations are so dominant that even the superposition of direct and steric potentials interaction fails to describe the interaction [24]. However, the scaling behavior of the damping rate given by (16) and (17) should still hold, provided one uses the fully renormalized $l_{0}$ and $\xi$.

So far, the membrane has been described with the traditional picture. Even without an explicit calculation, one would expect that including the bilayer aspects would lead to a second slow mode at small $q$ with $q^{2}$ dependence, corresponding to the slipping mode $\gamma_{2}$ in (12). If their frequencies become comparable at larger $q$, the modes will mix as they do for the free membrane.

\section{Dynamics of a bound polymerized membrane}

The theory reviewed here can also be applied to polymerized membranes [1], provided two conditions are met: (i) the membrane is impermeable to the fluid, and (ii) the membrane can still be considered as 
incompressible. Both conditions hold for the compound red blood cell membrane as well as for phospholipids in the gel phase. The crucial difference between polymerized and fluid membranes arises from the renormalization of the bending rigidity by the coupling between the in-plane phonons and out-of-plane shape fluctuations [30]. For free membranes this effect leads to a crossover length $\xi_{*}$ separating fluid behavior on small length scales from polymerized behavior on long length scales. Consequently, the energy $E(q, \xi)$ of a bound polymerized membrane [31] entering the expression (16) for the damping rate exhibits three scaling regimes:

$E(q, \xi) \approx \begin{cases}\kappa \xi_{*}^{2 \zeta-2} \xi^{-(2+2 \zeta)}, & q \ll 1 / \xi, \\ \kappa q^{2+2 \zeta} \xi_{*}^{2 \zeta-2}, & 1 / \xi \ll q \ll 1 / \xi_{*}, \\ \kappa q^{4}, & 1 / \xi_{*} \ll q,\end{cases}$

where $\zeta \simeq 0.5-0.65$ is the roughness exponent. In (23), it is implicitly assumed that the potential is weak enough for the polymerized behavior to show up in an intermediate range, i.e. we assume $\xi>\xi_{*}$. For strong potentials, one would expect that the fluctuations are so confined that the intermediate regime in (23) is missing. In this case, the polymerized membrane will behave like a fluid one.

Thus, for small $q$, the damping rate is again given by the universal $q^{2}$ dependence. With increasing $q$, there are a whole set of possible crossover scenarios depending on the relative size of the three length scales $l_{0}, \xi$, and $\xi_{*}$. For example, consider the typical case $\xi_{*}<l_{0}<\xi$. Then $\gamma \sim q^{2}$ crosses over at $q=1 / \xi$ to $\gamma \sim q^{4+2 \zeta}$, at $q=1 / l_{0}$ to $\gamma \sim q^{1+2 \zeta}$, and finally at $q=1 / \xi_{*}$ to $\gamma \sim q^{3}$. The behavior in the two intermediate cases has been found previously for two parallel polymerized sheets by Frey and Nelson [32], while the free behavior $\gamma \sim q^{1+2 \zeta}$ has also been obtained by simple scaling [33]. For $l_{0}>\xi$, one can again obtain nonmonotonic behavior with $\gamma \sim 1 / q$ in an intermediate $q$ range.

\section{Acknowledgement}

We thank the organizers for the opportunity to present this work at such an exciting workshop. Stimulating discussions with E. Evans, W. Fenzl, S. König, R. Lipowsky, J. Rädler, D. Richter, E. Sackmann, H. Strey and M. Wortis are also gratefully acknowledged.

\section{References}

1 Structure and conformation of amphiphilic membranes, eds. R. Lipowsky, D. Richter and K. Kremer (Springer, Berlin, 1992). R. Lipowsky, Nature 349 (1991) 475.

2 L. Kramer, J. Chem. Phys. 55 (1971) 2097.

3 F. Brochard and J.F. Lennon, J. Phys. (Paris) 11 (1975) 1035.

4 U. Seifert and S. Langer, Europhys. Letters 23 (1993) 71.

5 U. Seifert, L. Miao, H.G. Döbereiner and M. Wortis, in: Structure and conformation of amphiphilic membranes, eds. R. Lipowsky, D. Richter and K. Kremer (Springer, Berlin, 1992) p. 93.

6 W. Wiese, W. Harbich and W. Helfrich, J. Phys. Condens Matter 4 (1992) 1647.

7 R. Waugh, J. Song, S. Svetina and B. Žekš, Biophys. J. 61 (1992) 974.

8 E. Evans, A. Yeung, R. Waugh and J. Song, in: Structure and conformation of amphiphilic membranes, eds. R. Lipowsky, D. Richter and K. Kremer (Springer, Berlin, 1992) p. 148.

9 W. Pfeiffer, S. König, J.F. Legrand, T. Bayerl, D. Richter and E. Sackmann, Europhys. Letters 23 (1993) 457.

10 E. Evans, Colloids Surfaces 43 (1989) 227.

11 U. Seifert and R. Lipowsky, Phys. Rev. A42 (1990) 4768.

12 R. Lipowsky and U. Seifert, Langmuir 7 (1991) 1867.

13 A. Zilker, H. Engelhardt and E. Sackmann, J. Phys. (Paris) 48 (1987) 2139. 
14 A. Zilker, M. Ziegler and E. Sackmann, Phys. Rev. A46 (1992) 7998.

15 J. Rädler and E. Sackmann, Langmuir 8 (1992) 848.

16 U. Seifert, Dynamics of a bound membrane, preprint.

17 Y. Marathe and S. Ramaswamy, Europhys. Letters 8 (1989) 581.

18 R. Merkel, E. Sackmann and E. Evans, J. Phys. (Paris) 50 (1989) 1535.

19 H. Engelhardt, H.P. Duwe and E. Sackmann, J. Phys. Lettres 46 (1985) L395.

20 I. Bivas, P. Hanusse, P. Bothorel, J. Lallane and O. Aguerre-Chariol, J. Phys. (Paris) 48 (1987) 855.

21 S. Milner and S. Safran, Phys. Rev. A36 (1987) 4371.

22 F. Nallet, D. Roux and J. Prost, J. Phys. (Paris) 50 (1989) 3147.

23 F. Brochard and P.G. de Gennes, Pramana Suppl. 1 (1975) 1.

24 R. Lipowsky and S. Leibler, Phys. Rev. Letters 56 (1986) 2541.

25 S. Leibler and R. Lipowsky, Phys. Rev. B35 (1987) 7004.

26 R. Lipowsky, Physica Scripta T29 (1989) 259.

27 J.N. Israelachvili, Intermolecular and surface forces (Academic Press, New York, 1985).

28 M. Mutz and W. Helfrich, Phys, Rev, Letters 62 (1989) 2881.

29 W. Helfrich, Z. Naturforsch. 33a (1978) 305.

30 D.R. Nelson and L. Peliti, J. Phys. (Paris) 48 (1987) 1085.

31 R. Lipowsky and M. Girardet, Phys. Rev. Letters 65 (1990) 2893.

32 E. Frey and D.R. Nelson, J. Phys. I (Paris) 1 (1991) 1715.

33 R. Lipowsky, in: Festkörperprobleme/Advances in solid state physics, Vol. 32, ed. U. Rössler (Vieweg, Braunschweig, 1992) p. 19. 